\title{
WHAT IS A MEANINGFUL NAME? \\ Frege's idea of a radical semanto-ontological analysis
}

\author{
CONTENTS
}

\author{
1. Sense, meaning, and reference \\ 2. Realms of entities \\ 3. General theses concerning realms of meaningful names \\ 4. The forming of (abstract) names and objects \\ 5. Oblique contexts \\ 6. Formal truth and its pragmatic meaning
}




\section{Sense, meaning, and reference}

Some theories of language tell us that names have no meaning. A name just refers to the object named. On the other hand, we certainly may use the word „meaning” in the terminological way Frege uses the German word „Bedeutung". If we apply it to a name, the meaning is the object named; if we apply it to a 'standing sentence' or to an assertion, the meaning is the truth value of the sentence or the utterance. It would be probably a good idea to apply the word „meaning" also to predicates and other 'open' or 'functional' expressions when we want to refer to their 'extensions' ('courses-of value'), even if Frege himself did not, but this does not concern us here.

As we know, Frege differentiates between sense and meaning. The sense of an expression 'is' something like the implicit, 'empractical', way in which the meaning of the expression in question is determined. Despite the fact that we used the expressions "the sense" and „is" here, we shall see why in such a use we do not refer to any object, nor do we presuppose clear and distinct identity criteria for such senses. Rather, we 'point' to some 'experience' and 'practice' when we say, for example: The sense of an expression 'consists' in the criteria by which its meaning is determined. What it is to grasp and to master the sense or the criteria (or rather: their explanations) is learned and shown in common usage of the expressions. Things get complicated here, because in a developed (linguistic and cultural) practice many kinds of 'meta-level' or 'reflective' judgements and arguments belong to a usage of an expression as a whole. Think, for example, of devices like proofs by which we control the 'correctness' of object-level use, e.g. the 'truth' of statements.

There is no wonder, now, that an explanation of the concept of sense as given above cannot be read as a definition of how to use an expression like "the sense of the expression $E$ " in all linguistic contexts. It is rather a metaphorical hint or an illucidation for how we want to use the words „sense" in certain (not in all) meta-level contexts. Often we do not want much more than to shorten the expression "the way the meaning is determined" by the expression "sense": Just as there might be different ways leading different persons in different situations to the same result, the ('empractical') ways we learn and master the sense of an expression may differ, even if the meaning does not. What matters in (object level) language use is only that meaning, the 'result', is determined, not that there is a unique 
sense or 'way' leading to it. There might be no meaning without some sense. In other words: When we use expressions in (object level) sentences or statements, we do not reflect on their usage, nor do we think about any 'rule' of their use. Rather, we 'implicitly assume' that we already know how to handle the criteria defining the identity of meaning. By doing this, we do not presuppose criteria of identity of sense nor, in the case of 'rule-following', identities of rules. Naming senses (or rules or functions) is a metalevel enterprise.

Many modern theorists of language who see themeselves in the tradition of Frege's analysis go further and think that the sense of an expression 'is' something like a rule or a function. As possible arguments or inputs of such a function one could assume, then, $n$-tupels of 'things' (or 'entities') like the following: an expression taken as a linguistic form or type, a context, a generic ('abstract') and/or an individualized ('concrete') situation (turning the type of usage into a token of use), perhaps a speaker, a hearer, and perhaps other 'things' like interests or goals. A value or output of such a function could be an object if the expression was a name, or a truth value if it was a sentence. - We know the function or rule resp. the sense, it seems, if we know the values or outputs resulting from any of the possible inputs. But how can or do we know something like this? What is a function? How do we grasp a rule? These were some of the central questions of the later Wittgenstein. Indeed, these question are strongly related to the question how to grasp the sense of an expression and how its meaning is determined by this sense.

We may object against this idea claiming that 'formal languages' and 'mathematical functions' differ from 'natural languages' and senses respectively. We certainly have to distinguish between the ways in which the truth values (or truth conditions) of mathematical statements and other statements are defined. Nevertheless, a consideration of mathematical sentences can help us to show analogous features of other realms of talk and to see what it means to talk about object- and meta-levels of language-use.

According to Frege, not so much the senses of names, but concepts (predicates) 'are' functions. They are functions with exactly two possible values, called „(The) True” and „(The) False”. Their 'arguments' are objects, i.e. possible meanings of possible names.

It is important to see the following ambiguity: When we say that the meaning of an expression (i.e. of a saturated expression) is the object named, then there is a conventional reading and a rather unusual one. The first assumes that there are objects and that some expressions are used to name them. In this reading it seems more natural to translate the German 
„Bedeutung” by the English word „reference” or even „referent”. The second reading is this: What we call "objects" is constituted as the meaning of 'meaningful' expressions. We shall try to explain what this means. At first, however, some further remarks on the usual understanding of sense and meaning and some remarks concerning Frege's three realms of 'entities'

(?) are in order.

\section{Realms of entities}

In his explanation of sense and meaning, Frege uses a famous example: We refer to the planet Venus by different names like „hesperus" and „phosphorus". The meaning or referent is always the planet Venus.

Let us call certain linguistic representations of possible objects „names”, deictical 'presentations' (of possible objects) „appearances". Then we see: Different appearances of the planet Venus play a role in the sense of names like "the morning star" and "the evening star". I claim, now, that this example should be read as an analogy in the following sense: Like in the cases of physical objects where we can distinguish between the ways an object is represented and what the object itself is, we must distinguish between abstract objects like numbers and their different representations.

Once again, it is not much more than a façon de parler to say that the sense of the names "hesperus", „morning star" etc. is the way in which the reference of the names is given. Expressions like „the sense of "hesperus" ” or "the way Venus is named" are no meaningful names at all — just as an expression like "the function $x+y$ " does not name a function, as Frege says many times. We shall see later how we must construct the special contexts and special identity conditions when we want to turn 'senses' and 'functions' into well defined 'objects' of our talk, or, what amounts to the same, when we want to use an expression like „the sense of $X$ " as a meaningful name.

Frege was very aware of the fact that we sometimes use expressions that syntactically look like names or definite descriptions in ways that are to be distinguished from the use of normal, meaningful names. Hence, we should not assume that any senseful (or reasonable) use of a syntactical name already presupposes that the name is meaningful, i.e. that it names an entity or object in an already well constituted realm of objects.

If we take this into account, the following reading does not seem to be far off from Frege's writings: We do not have to assume strange 'ontic' realms of 'entities' like impressions and sensations, functions, senses and thoughts, abstract objects and concrete things when we want to distinguish between 
different uses of namelike expressions. We use titles as those listed above to distinguish between different ways to use such expressions. In short, I claim that Frege did not indulge in 'ugly' ontological dogmatics when he talked about three realms of 'objects', but rather made an important distinction between different uses of namelike expressions.

Let us assume, first, that the use of names for physical bodies is more or less well understood. Then we do not have to care too much about the 'constitution' of what Frege calls the first realm of objective and sensible 'entities': the realm of physical things. We shall, however, show below that and why a theory of names better is not too blue-eyed about concrete objects.

There is a second realm Frege did not care too much about, namely the realm of sensations, of feelings and other kinds of 'subjective representations'. ${ }^{1}$ This realm is not objective in the sense that there are no transsubjective identity criteria for particular sensations ('sense data') or for particular 'subjective pictures' (e.g. in my dreams). There are no controllable 'objective' identity statements about such sensations and feelings. Nevertheless, Frege did not and we do not have to deny that we can 'refer' or 'talk about' sensations or feelings in some way. We distinguish, for example, certain kinds of pain. What Frege denied is that there are individualized entities in this realm and that they can be the meaning of names. I think, he is right. And I think, much of Wittgenstein's later work develops these ideas of Frege, e.g. when he analyses the concept of pain. The expression „my headache" is, for example, syntactically a well formed name or "definite description'. But it is no meaningful name, at least as long as there is no truth condition defined for equalities of the kind „my headache now is the same as (yours) yesterday".

The other direction is even more important: Meanings of names are no entities in a 'mental' or 'psychological' realm. Meaning is and must be 'transsubjective'. This holds especially for abstract entities like numbers and sets: they do not belong to the second realm of sensible but not objective 'entities'.

Abstract objects form a third realm, the realm of objective, but non-sensible entities. There is no question (not only for Frege) that we can talk about such objects in a way such that there are 'objective' criteria defining the truth and falsity of the statements. In mathematics we talk about numbers, sets or ideal geometrical forms. Just because there are clear (formal) truth conditions defined for sentences (or utterances) that contain

1 The German word „Vorstellung” usually means subjective, mental (re)presentation, whereas „Darstellung” represents the objective, transubjective meaning of Latin „(re)presentatio". 
meaningful names, the 'existence' of the abstract objects to which the names refer cannot be questioned.

\section{General theses concerning realms of meaningful names}

Some say that Frege's analysis of singular terms is outdated by Russell's famous analysis of definite descriptions in On Denoting or by Kripke's criticism of all 'cluster theories' of meaning or reference and his differentiations between rigid and nonrigid designators in Naming and Necessity. But neither Russell nor Kripke answered the question how a realm of possible denotations is constituted. Like later semanticists Russell assumed that structured realms of objects were already given. The questions that neither he nor Post-Tarskian semanticists answered are these: What does it mean to say that an 'interpretation' turns formulas (sentence forms) into true or false sentences? How is a concrete 'model structures' really defined as it is used in model theoretical semantics when one wants to define formal interpretations? How are concrete sets and structures constituted in which (parts of) formal, axiomatized, set theory become 'true' statements? Usually, axiom systems only define classes of models ${ }^{2}$; they do not define what a concrete, particular, model is. Tarski and his followers never told us what a particular evaluation of a variable in a particular infinite interpretation-structure satisfying certain formal axioms and axiomatized theories is. The reason is that any concrete evaluation of a variable in a particular interpretation can only be a name or a representation of an (abstract) object. It can never be the object itself, at least not without a mediation of such a name. If one sees this, it becomes clear that Tarski's general model theory only talks about forms of evaluations and interpretations. I know that this seems to be a 'big' claim; if model theory is understood, I rather hold it as self-evident. One of the reasons for a restriction to formal semantics is the belief that one cannot define uncountable sets by saying how to deal with 'names' of their elements. Allegedly, a system of 'names' is always countable. But can model theory really be defined in the framework of axiomatized set theory? And what would it be to 'believe' in 'naive' set and model theory? We are not satisfied with such beliefs, because they mean not less than a lack of analysis. $^{3}$

\footnotetext{
${ }^{2}$ Notice that a set, as we should use the word, is always a formal object in a well constituted realm of objects we can talk and quantify about. The words „class” or „system" are used empractically, here, without presupposing „set abstraction”, as formal set constitution is often called.

${ }^{3}$ In this respect, the critical developments of Frege's ideas in the work of Prof. Paul
} 
If we ask what there is and what we can and do quantify about, a classical answer gives us the advice to believe in realms of possible 'entities' that can be values of variables. Such a belief is often called "ontological commitment". Logicians like Quine obviously assume that there are functions that lead us from variables or names as arguments to entities or objects as values. But what does it mean to assume that a function like this exists? How is such a function defined? What is it to be a possible value of a variable? The question leads us back to the questions above.

The reason why I am not satisfied with the answers or explanations that are usually given to these questions is this: They do not tell us what it means to believe in certain realms of objects or entities or to embrace certain ontological commitments. They do not tell us what functions or relations and objects really are or how they can be defined and identified. To see the difficulty, notice how superficial it is to assume abstract objects as given and to look for a relation between such objects and names. What is needed here is a constitutional explanation of particular realms of abstract objects. I even dare to claim that neither Quine nor Tarski nor any other modern 'formal ontologist' gave us a sufficient answer to this question. Instead, they made their different appeals to 'beliefs' and 'commitments'. Of course, they tried to overcome the mere appeal to vague beliefs by a precise axiomatization of a holistic framework like formal set and model theory. The concepts of formal truth and interpretations are defined in such a framework.

I cannot show all the problems of such a formalist theory of meaning and truth or of a philosophy of language following the formal lines drawn by Davidson and Montague. I just show some problems. In short, I claim that the procedure of formalist semantics is unsatisfying not because it is false, but because it stops short of asking and answering important questions. The most important is: What do we presuppose as clear when we use a certain formalistic framework? For it is crucial to see that any formalist analysis, e.g. any 'axiomatic foundation' of set and model theory or of mathematics, always leaves certain questions of content open. When we restrict our attention to what can be treated schematically, we always (and deliberately) avoid the disturbing fact that we would have to rely on reasonable judgement - a very vague concept - when we would have to answer questions concerning our reasons for the choice of the formal framework as a whole and questions about their right external use. This external usage takes place in contexts and situations in which we are interested in good orientations

Lorenzen and Friedrich Kambartel seem to be underestimated until today, even if some particular ideas of their 'constructivism' might be problematic. 
and successfull practices. The mastery of the internal rules of the formal games, e.g. of proofs controlling the correctness of our rule following, plays a certain limited role here. Of course, it is legitimate that in our division of labour 'working mathematicians' and 'technical logicians' are not interested 'professionally' in such 'external' questions. But it would be (and somehow already is) a disaster for a reasonable development of science if departmental research forgets that not (only) the internal, regional, criteria of 'success' determine the reasonability of the research itself, but the place of the research and its results in a division of scientific, technical and cultural labour.

Different from a merely formalistic procedure, Frege wants to show how (structured) realms of objects can be and are constituted, namely via a realm of possible 'names' and by an explanation of truth conditions for equalities and other sentences. Frege knows that there is no entity and no name without reference to a whole system of names and sentences. As long as there is no analysis of the linguistic constitution of the realms of entities, we remain naive and do not even know what we are committed to and what it is to believe that quantified sentences were meaningful ('true' or 'false').

The question how a realm of objects is constituted does not merely arise in the case of abstract objects. Neither abstract, nor 'concrete' objects are just (to be treated as) 'given entities'. The question what a particular object in such a realm of objects is cannot be answered sufficiently by naming or showing the object 'immediately'. Names and examples give us only (re)presentations of such objects - just like any presentation of a number is a presentation of a name or of another 'representation' of the number. This is so, even if the object is a concrete thing like a chair or a table. In some sense, nobody can really 'show' us a thing like a chair as such. What can be shown are always (re)presentations of the thing. At least for such objects, linguistic expressions (names) are only a special kind of representations; appearances are another, especially if they are accompanied by situation-dependent utterances of namelike 'descriptions' as, for example, „this object here".

There are presupposed criteria telling us which sensational and symbolic representations (would) count as representations of the same object or, what amounts to the same, which representations are to be treated as 'equivalent'. Only by such (truth) conditions for equalities it is defined what the representations are representations of. Notice that there are 'phenomenological' forms of talking 'about' sensations or gestalts or subjective representations without already referring to physical objects. Only 'later' objects can be counted as bodily causes for 'sensations'. 
On a reflective meta-level we can try to show and even name criteria 'lying behind' our use of representations as 'representations of the same object'. The same holds for the criteria used in differentiating between 'representations of different objects'. It might not have been a bad idea when Hegel, following Spinoza (and Leibniz), tried to express the relation between these two groups of criteria by formulas that could be rephrased like this: Identity 'is' equivalence and equivalence 'is' non-distinction. Identity (of objects) involves an 'inner' or 'hidden' negation ('renouncement') of (possible) 'negation' (differentiation).

Again we should distinguish between the use of representations 'as representation of objects' and the reflective constitutional ('transcendental') analysis of the equivalence relation presupposed in such a practice.

Now we may understand in what sense we can say: A concrete thing, taken as the reference of a name, is not just there. At least its identity in space and time is presupposed as defined by criteria, by equality-conditions - where space and time should be seen as a framework of projection rules coordinating the 'objective space-time-references' of different (possible) observers. Such remarks on the constitution of physical objects are also directed against the idea that a thourough analysis of abstraction could start with physical objects and their properties and reduce the talk about abstract objects in one way or other to statements about (classes of) concrete physical things. Such a reductionist theory of abstraction is 'metaphysical' and 'dogmatic' insofar as it presupposes not only the way we talk about the physical world as unproblematic but it assumes that the concepts of physical object, physical truth, and physical 'reality' were independent of our way we represent and deal with them. This shows, in the end, that and why any ontic or epistemic physicalism is a metaphysical dogma, a modern 'religious' belief binding the horizon of physicalistic and reductionist thinking.

There is no well defined realm $R$ of objects or entities without a presupposed set of criteria that tell us

a) what counts as a possible representation of such an object, i.e. what belongs to the categorical realm $\boldsymbol{R}$ of possible $R$-representations and

b) which different (re)presentations count as (re-)presentations of the same $R$-object or entity.

Names are a special sort of symbolic representations. When we ask, which pairs of (re)presentations, names or appearances, are names or (re)presentations of the same object, we normally assume that the representations already belong to the same 'category'. We call it $\boldsymbol{R}$. Such a category $\boldsymbol{R}$ of (re)presentations (names or appearances) is an important part of a constitutive definition of a realm $R$ of objects we want to talk about. 
We have to distinguish, now, between mere 'syntactical' names of a certain category $\boldsymbol{R}$ and semantically well formed or 'meaningful' names of the category $\boldsymbol{R}$. In a similar way we distinguish between formally or syntactically well formed sentences and 'meaningful' sentences (belonging to $\boldsymbol{R}$ ).

Let us assume that a certain class $F N_{R}$ of expressions that are formally categorized as singular terms ('formal names') of category $\boldsymbol{R}$ is determined by a lexicon and by syntactical rules of name-formation. And let us assume that further conditions must be fulfilled by elements of $F N_{R}$ in order to belong to a class $N_{R}$ of semantically meaningful singular terms ('names') of category $\boldsymbol{R}$. Not all expression of $F N_{R}$, only expressions of $N_{R}$ may occur in (meaningful) contexts of the category $\boldsymbol{R}$. Let us call the class of these contexts $C_{R}$.

To look at a more concrete example, let $F N_{R}$ be the class of expressions of the form „,n/m”, where $n, m$ are (meaningful) names of whole numbers. (We assume that the conditions for being such a number term are clear.) Let $N_{R}$ be the class of expressions, in which $m=0$ is false. Let $C_{R}$ the context of all sentence(-forms) that belong to our 'talk about rational numbers'. An example is: , $5 / 3+1 / 9=16 / 9 "$; counterexamples are:,$n / m$ is a ratio" or „2/4 is another fraction than $1 / 2$ ". Statements like these do not belong to $C_{R}$ despite that fact that the two fractions are equivalent representations of the same ratio and the same rational number.

When the meaning or reference depends on the context and situation of the utterance, we have to replace „meaningful names” and „meaningful sentences” by something like „meaningful utterance of an expression belonging to the category of singular term-utterances resp. of statements". A name or sentence may be 'meaningful in general', even if it is meaningless in certain contexts and situations of utterance. We do not consider this more complicated situation here, even if it is important to see that it also appears in mathematical discourse, for example when we talk about elements of 'uncountable' sets that are not named in a prefixed system of possible names. Think, for example, of an expression that 'describes' a diagonal in Cantor's diagonalization procedure.

We cannot add up all categories to one overall category of all names or all well formed singular terms. We also cannot add up all realms of objects to one overall ontological universe of discourse. Hence, we cannot re-define particular categories or realms of objects by predicates that sort them out of a 'universal ontology'. I do not go into the reasons, here, why this is not possible. I only mention that in order to follow Frege's main idea we have to depart from some of his particular proposals. The difference is this: A name is a name only with respect to a special context $C_{R}$. The description 
of $C_{R}$ is an important part of an analysis of the 'semantical category' to which the names and objects and object-level statements belong. 'Category mistakes' (like , $2+2$ is a candle") have to do with our "semantical categories'. Such semantical categories are to be distinguished from syntactical categories labelled „names”, „sentences” (and so on) as they are analysed by Ajdukiewicz and play important roles in formal 'categorical grammar'.

A word belongs to the syntactical category 'name' when it 'may' appear at certain places in certain sentences or in other complex expressions (of a certain syntactical category). Syntactically complex names are defined by certain syntactical devices. A general syntactical form of a complex name is that of a definite description: „the $x$ that has the property $E$ ". But there is one important caveat already to mention here: Not every (meaningful) syntactically complex name can be treated semantically as a 'definite description', even if it should look like a definite description. In other words, we should define a definite description by the semantical way its use is defined and not by the syntactical form.

The importance of the last remark will become clear if we consider the basic ideas of Frege's Basic Laws of Arithmetics. Contrary to many readings, Frege treats terms of the form ,the course of value of the function $f(x)$ " (or rather the corresponding formal expressions) not as definite descriptions. Rather, he treats them as semantically basic names. What does this mean? Well, it means that the constitutional definition of the 'meaning' of such an expression differs considerably from the definition of the meaning of a definite description. Before I explain these differences more precisely, I would like to give them labels ('names'). I.e. I want to distinguish between (at least) two kinds of syntactically complex names, namely definite descriptions that are syntactically and semantically complex names, and non-elementary basic names that are syntactically complex but semantically basic with respect to a corresponding semantic category $C_{R}$. Kripke does not see how an expression like the above can be a semantically basic name instead of a description. Indeed, what is a semantically basic name?

Before I try to answer the last question, I go back to the question of our title. On the ground of a mere formal categorization of expressions as names (or as namelike), the following question certainly makes sense: Which syntactical (elementary or complex) names are meaningful in the sense that they denote something? After we (think we) have an answer to this question, we usually use the word "name" in the comprehensive sense of 'meaningful name' or in the sense of 'meaningful utterance of a namelike expression'. Sometimes, however, we use it in the sense of 'meaningful elementary name', 
or in the sense of 'meaningful basic name'. If we adopt one of the latter conventions, definite descriptions are no names at all.

I think that any understanding of Frege's enterprise has to answer the question: What comes logically and methodolically first, the syntactical names, the real symbols and real '(re)presentations', or the 'semantical meaning' (whatever it may be), i.e. the object named? This is not a question of the sort: Was the egg first or the hen? It is a question of the sort: How is the meaning or the reference of an (uttered) expression (name or sentence) defined?

I claim that there can be only one satisfying answer: We must start with the (re)presentations before we can assume that we know what the objects are that are represented and before we 'believe' in the 'existence' of such objects (whatever it is to believe such things). Kant, by the way, was the first to see the importance of this principle with respect to the constitution of the meaning of expressions that name 'physical things', whereas Frege tried to apply the same principle in a constitutional analysis of the concepts of numbers and sets.

Frege's seemingly strange statement that the meaning of a name is the object named now can be read as the proposal to define the objects in their identity by the truth conditions of sentences, especially of equalities, in which one or more names of the object occur. Proceeding this way we can avoid unanalysed metaphysical beliefs in allegedly pregiven entities or realms of objects. What is pregiven is a form of practice and a form of speech. This and nothing else is the core of a real linguistic-pragmatic turn of transcendental analysis. Thus, transcendental analysis it turned into an analysis of presuppositions, as it is shown by Strawson and many others.

Frege's famous 'holistic' claim that a name has meaning only in the context of sentences (I read the German phrase ,im Satz” generical) is an important part of such an analysis: Something is an object (a nameable entity) only with respect to a certain system (network, category) of properties and propositions. The properties and propositions are not only expressed but constituted by 'rules' for the use of forms of sentences or utterances in which the expression (name) can occur meaningfully.

We can be a little more precise: An expression is a (formally) meaningful name with respect to a given system of contexts or sentences, if all the sentences of the system, in which the name occurs, are (formally) meaningful. Sentences (utterances) are (formally) meaningful if their truth conditions are defined. Notice, however, that there are further properties that systems of sentences have to have in order to turn a system of possible names into a system of (formally) meaningful names. We shall come to this immediately. 


\section{The forming of (abstract) names and objects}

We speak about abstract objects when the formal truth conditions for sentences containing symbolic or linguistic representations are defined by 'rules' that do not depend on non-symbolic appearances of the objects. Hence, it is a conceptual truth that we cannot 'see' or 'feel' abstract objects, and that they are no 'impressions in our mind' (whatever the last expression should mean). Abstract truths as such are not tested immediately by experience. They are defined by conventional rules we have to learn and we can learn just by listening to texts, reading books, and by learning to rephrase and formally deduce further sentences. To show how we can analyse abstract objects of a certain category, we consider the example of (natural) numbers. We have to answer the following questions:

1) What are (syntactically well defined) candidates for meaningful names of numbers?

2) What is a meaningful name of a number?

3) How are the truth conditions for equalities between meaningful names defined?

4) Which further properties (sentence-forms with truth conditions) are defined for the numbers? I.e. what sentence-forms are to be taken in consideration and what are their satisfaction conditions?

As we said already, a name $N$ always presupposes a whole system of names and a whole system of sentences $\mathrm{S}$ including equalities of the form "N $=M$ " such that for the sentences the following conditions hold:

5) For any sentence of the system there is one and only one of two truth values (e.g. 1 and 0 , or $T$ and $F$, or True and False) defined. (This principle could be called the principle of distinction or consistency.)

6) If the value of an equality $N=M$ is the peculiar value called "True", and if the value of $S(N)$ is "True", then the value of $S(N / M)$ is "True". (This principle can be called the principle of Leibniz.)

We can say, roughly, that if 5.) and 6.) are fulfilled, a one place sentence-form (or predicate) "S(x)" divides the names (or the objects named) into two classes. On the other side, a name (an object) divides the sentence-forms (predicates) into two classes. The principle of Leibniz 'says' that the distinctions defined by the predicates are not (or may not be) finer than the equation (or equivalence) $N=M$. True equalities 'say' that in the corresponding semantical category that we do not (and cannot) differentiate between possibly different but equivalent representations (names or appearances). The last means that they 'have the same meaning' and 'name the same object' with respect to $C_{R}$. 
The condition of consistency is not fulfilled, if for some sentences " $S(N)$ " the accepted or presupposed criteria "say' that „S(N)" is valued as true and at the same time it 'is' (valued as) false. If we would express this on the 'object-level', we would have to say something 'nonsensical' like: Some entities $N$ 'do have and do not have the property $S(x)$ '. If we know what a sentence like this 'really' says, we even may use it. It means that ",S(x)" is no admissible predicate for $N$ or that " $N$ " is no admissible name resp. $N$ is no admissible object for ",S(x)".

Another, though similar, 'dilemma' arises if for a sentence „S $S(N)$ ” no truth condition is defined. A standard remedy seems to be, then, to attach the value "False" to it. This seems to be a convincing strategy, because we may think that what is not true by our truth criteria is just false. It is, however, a dangerous assumption or strategy. In fact, it was this assumption that ultimately lead to Frege's mischief in his Basic Laws of Arithmetics, namely to Russell's paradox. The liar's paradox, too, results from this dangerous, or rather wrong, presupposition concerning the criteria for truth and falsehood. I do not say more to this here, even if more than a moments reflection is needed to see what is said by these claims. Rather, I present another small model in order to show how the constitution of a realm of objects and a realm of properties is to be understood and what we have to do in order to turn expressions into formally meaningful names and sentences.

Our example is a short story. Its persons are Josef, Joe, Fred, Jim, Caruso, Ralph, the sailor. Well, I rather should not use object-level expressions. I rather should say: the candidates for (basic) names that shall appear in my (retelling the) story are: „Josef”, „Joe”, „Fred”, „Jim”, „Caruso”, „Ralph", „the sailor". The story goes like this: Fred hates Caruso. Jim hates Fred. Joe loves Fred. Ralph loves Fred and Josef. The sailor loves Joe. Joe kills Joseph. The story tells us, further, that Fred is called "Caruso" and Joseph is called „Joe”.

We know now that our story does not speak about 7 but about 4 (or about 5) objects ('persons'), namely Joe, Fred, Jim, Ralph, and, as we shall see, possibly about a fifth person called „the sailor”. The only relations that appeared in our story were love, hatred and killing - if we neglect identity. So far, our story does not present us a 'complete picture' of 'all relations' between the persons.

There are standard logical principles by which we usually 'enlarge' or even 'complete' such stories. We do this by adding ('implicitly') more sentences than what we really read. Not only the sentences we really read or hear but the added sentences, too, define the 'properties' (one place statements) and 'relations' (i.e. relational statements) between the 'objects' our 
story talks about. We usually use rules of the following sorts: For making our story a story 'about' persons or objects, we use the Leibniz-Principle (LP), which defines equality: If $A(N)$ is true and if $N=M$, then $A(M)$ is true. Some terminological rules (TR) are usually presupposed, too: If $N$ loves $M$, then $N$ does not hate $M$. If $N$ hates $M$, then $N$ does not love $M$. In order not to complicate things too much here, the story does not contain logically complex, e.g. quantified, sentences. But it is clear that such sentences are used by applying the rules of predicate calculus (PC). (These rules often include LP).

In our example we use LP in order to conclude that Joe commits suicide. Because we say that to commit suicide is no murder (this is a terminological rule belonging to type TR), we can apply a rule of PC to get the following result: there is (possibly) no murder(er). The story does not tell us that any person killed another person. Hence, the expression "the murderer", if we read it as a definite description, is not meaningful, does not have a meaning, does not refer to anybody with respect to the story.

When we apply rules like these we often say that the consequences 'follow logically resp. conceptually' from what was said in the story. For some readers, even the following rule might be assumed: "N always loves $N$ ". This, however, is rather a 'synthetic assumption' (SA) than a terminological rule.

In the end, a reader might even assume the following rule: „If our story (together with a use of 'rules' listed above) does not tell us anything about the relation of love (resp. hatred, killing and even identity) between $N$ and $M$, then we may assume that $N$ does not love (hate, kill, or is equal with) $M$. We call an 'assumption' like this a 'completeness rule' (CR) with respect to the set of elementary (i.e. logically non-complex) sentences defining the possible properties $S(x)$ and relations $S(x, y, \ldots)$ of the categorical context-system $C_{R}$. CR presupposes that we know the whole story, especially that we know all relevant contexts of category $C_{R}$. When we 'concluded' that according to our story there is no murderer, we already used CR silently.

When using CR, positive elementary sentences play a special role: If we cannot deduce the positive sentences from the text by rules of the first three types, CR tells us to assume the negated assertions. Things get complicated if the text contains more logically complex statements and if the story is (to be) treated as an uncomplete picture. Indeed, it is often unclear how far we can rely on rule CR: We might read the story as a mere beginning of a longer story. I claim, however, that we always use rules or principles of the first four types, and that we often use CR, too, when we 'understand' a 
text. Even when we say what the story says 'explicitly', we normally do not restrict our judgements to sentences that are listed explicitly.

This means that we do not read such texts merely as axiomatic systems 'describing' classes of possible structures on arbitrary sets of objects that only have to have 'enough' elements in order to fulfill the formal conditions of the story. We often assume silently that a story describes a structure that is 'smallest' and 'easiest' in the sense that it does not contain more objects or elements and it does not contain more true elementary statements about them than it must in order to fulfill the conditions told in the story. Often, of course, such a 'smallest' and 'easiest' structure is not determined uniquely.

Even if a story only describes 'possibilities', i.e. if it only determines a subclass of 'really' possible state of affairs in a full class of 'all' possibilities, any such possibility is defined by a possible 'full description' of a relational structure or model.

If we use 'full' rule CR together with the other rules, the class of 'really' possible state of affairs defined by our story contains exactly one structure, the 'real state of affairs' of the story - if we restrict our attention to the objects named in the story. With respect to this state, we can answer 'all possible $C_{R}$-questions' concerning what is true about our persons.

If rule CR is omitted or weakened, the story only tells us, which worlds are the possible worlds according to our story. Notice that to define one of these structures by the truth values of the corresponding elementary sentences of a categorical context-system $C_{R}$ is something else than to define subclasses in a class of 'all possible' structures by an axiomatic theory, i.e. by a list of logically complex formulas and the rules of PC. There is no way to describe a particular formal structure in another way than by describing the true and false sentences 'of it'. This means that the usual proceeding of set theoretic model theory already presupposes the class of possible models or, what amounts to the same, what it means to quantify over all sets of naive set theory. ${ }^{4}$

With respect to our (formal) real state of affairs, some definite descriptions of the form "the $x$ such that $A(x)$ " are meaningful, others are not. „The person loved by Joe" is a meaningful definite description (assuming $\mathrm{CR}$ ), „the murderer” is not. But for all such expressions, their sense is defined.

In On Denoting, Russell reformulated the idea of Frege how to use definite descriptions $t$ with respect to a realm $R$ in sentences $S(t)$ of a context-

\footnotetext{
${ }^{4}$ I do not say anything, here, about a mere internal model theory that does not leave the framework of formalistic, axiomatized, set theory, even if this would be important for an analysis of Tarski's work.
} 
-system $C_{R}$. In Frege's analysis (as I read it by relativizing it to a category $\boldsymbol{R}$ and a realm $R$ of objects), a descriptive term $t$ has to have the form "the $x$ such that it has the property $A(x)$ " where $A(x)$ is itself a context of $C_{R}$. Such a definite description $t$ is meaningful with respect to a realm of objects $R$ if and only if the well known existence (1) and uniqueness (2) conditions are fulfilled: (1) There is an object in $R$ named by a possible meaningful (basic) name $N$ such that $A(N)$ is true and (2) for all other objects named by possible names $M$ (for which $M=N$ is false) $A(M)$ is false. (Notice, again, that such a basic 'name' $N$ may be a meaningful utterance of a namelike expression like "this tree in front of my window" or "this hand here". An utterance like this is not always to be read as a definite description. It can be used as a basic name. In other words, basic names do not have to be just elements of a system of syntactically defined names.)

Russell's analysis proceeds differently, namely by declaring all (object-level) formulas of the following form $(*)$ as 'axioms':

$$
S(t) \leftrightarrow \text { there is an } x:[A(x) \text { and for all } y: A(y) \rightarrow x=y \text { and } S(x)]
$$

If we leave a mere formalistic (axiomatizistic) framework and read the axioms $(*)$ as principles to determine the truth condition of sentences $S(t)$ with respect to a structure $R$, the result of $(*)$ is: A definite description $t$ is meaningful in Frege's sense if and only if there is at least one true sentence $S(t)$ of $C_{R}$ in which $t$ occurs. This holds if an only if for any elementary (hence for all logically complex) sentence-form $S(x)$ of the appropriate realm $C_{R}$ of discourse either $S(t)$ or non- $S(t)$ is (valued as) true ('and not both') in $R$.

In other words, if $t$ is a definite description with respect to a fixed realm $R$, the truth conditions of Russell's procedure in On Denoting tell us that for some (hence for all) properties $S(x)$ in $\boldsymbol{R}$ the statements $S(t)$ and non-S(t) are both (valued as) false if and only if t is not meaningful in Frege's sense.

The result is a certain equivalence between Frege's and Russell's analysis: A definite description $t$ is meaningful if and only if it 'behaves like' a meaningful basic name of the realm $R$ of our discourse. For an expression $E$ to behave like a basic name means: For any sentence-form $S(x)$ of $\boldsymbol{R}$ the sentence $S(E)$ has meaning, i.e it is (defined as) true or false (not as both).

But let us come back to our story, now. When we do not use rule CR and read the text as a mere beginning of a possibly longer story we might assume that Ralph is the sailor, 'because' he loves Joe, and the sailor loves Joe. If we assume this, we would turn the expressions „the one who loves Joe" and "the sailor" into meaningful descriptions with respect to the four persons named by 'Christian' names like "Ralph” and „Joe”. But we can read "the sailor", too, as a basic name, as I try to show now. 
With respect to the realm of objects given in our story, a new namelike expression like „Peter" does neither have sense nor meaning, whereas an expression like "the sailor" or "the murderer" seems to have sense, even if the story does not fix their meanings. (Notice that this is not a question of knowledge.) What do we have to do in order to make Peter into a person in our story? Just to add the name „Peter” to the list of elementary names does not suffice. We can turn "Peter" into a name of an object in our story only if some predicates (properties, relations) 'for Peter' are defined. We might, for example, use principle $\mathrm{CR}$ and assume that Peter is different from all others and neither loves nor hates nor kills anybody else in our realm. Then, Peter is a fifth person.

We might proceed, now, in the same way with the expression „the sailor”, considering what the story 'says explicitly' about a person with that 'nickname'. Then, the number of persons of our original story is not four, but five. Indeed, if we use CR in full scale, this would be 'the real' reading of our story: There is no way for reading „the sailor" as a definite description if we did not assume that our story were incomplete.

This short and certainly not sufficient example shows why Frege said that we cannot just 'construct' objects if not with reference to a whole system of objects. Hence, we cannot construct new abstract objects by a declaration like the following: Assume that the real equation,$x^{2}=-1$ " had a solution and name it , $i "$ or "the positive imaginary root of $-1 "$. Then you will arrive at the complex numbers.

The real procedure has to tell us what possible names of complex numbers are and what possible properties they have. And this must be done in a whole system. Hence, Frege does not contradict himself when he said that 'in some sense' his own procedure in the Basic Laws of Arithmetic is also constructive. The main difference to a 'naive' construction of objects by adding new names to a system of names are Frege's holistic principles that define what it means that a name is meaningful with respect to a context or category or 'story' $C_{R}$.

A definition of a realm of basic $R$-names occurring in elementary sentences and of truth values (or truth conditions) for $R$-sentences is the basis for a definition of the truth value or truth conditions of logically complex $R$-sentences in which sentential connectives and quantifiers occur. An implicit reference to a (possible) system of sentences fulfilling the principles listed above is the basis, too, for the interpretation of a deduction according to the rules of classical predicate calculus as a 'valid consequence'.

A common confusion in interpretations of Frege's enterprise results from the following mistake: Frege thought that we can combine any two realms 
$R, Q$ of objects just by adding two new predicates $R(x)$ and $Q(x)$ and a principle of the form: any object $N$ of the first realm $R$ is totally different from any object $M$ of the second realm $Q$. I.e., for any elementary sentence "S(x)" of the second realm ",S(N)" is wrong if ",R(N)" is true; and for any elementary sentence " $S(x)$ " of the first realm, "S(M)" is wrong if "Q $Q(M)$ " is true. This was the (wrong) reason why Frege thought that we did not need to bother about the local realms of the quantifiers but could combine all realms into one realm of 'all objects of meaningful discourse'. This is also the reason why he says in his Principles of Arithmetics that we have to make sure that a number, let us say the number five, is distinguished by some property from everything else, even from the sun and the moon. In other words, Frege did not see that the constitution of realms of entities as realms for qantificational logic always are bound to local categories.

\section{Oblique contexts}

If we talk about the sense of an expression in special 'oblique' contexts, e.g. in belief-contexts or in other contexts expressing 'propositional attitudes', we often presuppose some peculiar identity criteria for different representations of the same sense. Talking about the sense of an expression in such contexts has to be distinguished from using the word "sense" in order to 'point vaguely' to the implicitly known or presupposed criteria determining a 'normal' (non-oblique) 'use' of the names in sentences and the 'way' the truth or falsehood of the sentences or statements is determined.

We could express this differentiation thus: In oblique contexts we 'constitute' and 'talk about' new realms of objects called „the sense (or the meaning) of the expression $E$ ". In a similar way we talk about abstract entities called "the belief, the opinion, the propositions that $p$ " or the ,intention articulated, expressed or shown by the linguistic act $p$ " or "the intention lying behind the behaviour $b$ ". We do so by presupposing some new identity criteria for nominalized expressions and (possibly) some other truth conditions for utterances containing the expressions in question.

An expression of the form ,the sense of $X$ " is a nominalization. In oblique contexts, such a form is used on the ground of a certain implicit presupposition. What is presupposed is a kind of identity or rather: equivalence relation $\sim$ between possibly different expressions. We attribute to equivalent expressions 'the same sense' or 'the same belief' or 'the same intention'. We do this in our metalevel, reflective, talk, whereas in object-level contexts we presuppose the equivalence relation and we use the above expressions as 
names. - Unfortunately, Frege did not tell us enough about this constitution of oblique contexts. Therefore, the following statement may lead to some misunderstandings, if it is not read with a grain of salt: In oblique contexts, the meaning of an expression 'is' its normal sense. The objects we talk about in oblique contexts are closely related to the way the meanings of the expressions are determined in non-oblique usages of the expressions. The (presupposed) equivalence relation $\sim$ determines (implicitly) 'how close' this relation is. It depends heavily on the context in which we use the expression "the sense of $X$ " as a singular term.

If the meanings are different, the senses should be different, too. This principle says that any equivalence relation $\sim$ used for defining the sense of an expression as an object of talk in an oblique context should be finer than the equivalence relation defining identity of meaning in a corresponding object-level. In short, senses 'formally supervene' on meanings. We should see, however, that the differentiations between sense and meaning, between non-oblique and oblique contexts and between an object-level and meta-levels are, in a large extent, context-relative.

Expressions having the same meaning may be counted as expressing (or, in certain oblique context: naming) different senses. Which expressions, utterances or (symbolic or nonsymbolic) acts 'have the same sense', as we say, depends on many things, especially on the interest we take in the specific 'meta-level' and 'oblique' differentiations and identifications ('nondifferentiations') of senses, intensions, propositions, properties, intentions and so on.

\section{Formal truth and its pragmatic meaning}

Insofar as all texts in written books can be learned, they can in some sense always be read as 'abstract' texts about 'abstract' objects. Thus, 'formal knowledge', i.e. knowledge of literature, is largely independent of 'empirical' knowledge concerning 'concrete' things or phenomena. It often is not clear whether we talk about a concrete or about an abstract and ideal person or object when we talk about Moses, Homer, Socrates, or other persons of written history, or when we talk about atoms, electrons, quantums, neurons and other objects of scientific literature. Sometimes, we know fairly well that we do not talk about 'real' persons or objects, for example when we talk about Sherlock Holmes or about the 'personal God' of Jews, Christians or Muslims. On the other hand, historical or empirical truth is not the only 'good' reason we use when we orient our actions on sentences we assume as formally true. This can be seen clearly in the case of arithmetics and 
geometry, or if we regard certain functions of literary fiction or of religious beliefs. If we understand this basic formality of the concepts of truth and of the concept of an object we might see that the question is still open which criteria are presupposed in our distinctions between 'real reality' and superficial appearances, between 'concrete' and abstract ('theoretical', 'ideal') truths or between a mere story and 'true' history. And we might see that the relation between criteria telling us what we may count as reasonable knowledge and well corroborated certainty and what would count as 'real truth' in an infinite and ideal perspective 'sub specie aeternitatis' or for a 'possible god' is more complicated than 'realists' and 'physicalists' usually think.

The truth or falsehood of sentences (or statements) is, of course, in most cases not just a formal matter. We use true statements in further judgements and for an orientation of our actions. We do this not so much because only true statements will lead to personal (individual) success, but because our definitions of truth values or, what amounts to the same, our (generic) differentiation between true and false statements is already made in a way that it can and does play a specific role for (generic) differentiations between 'good' (e.g. 'probably successfull') and 'bad' (e.g. 'probably unsuccessfull') orientations. In mathematics, for example, we use only true (or rather: proven) mathematical statements as (allowed) rules in calculations and deductions. Of course, sometimes, false applications or applications of false rules might lead by chance to 'right' results. This is, of course, no counterargument against the advice that we should only use statements and rules for which we have shown that they fulfill the conditions defining their 'truth'.

Some philosophers of mathematics, however, believe that we only 'believe' in certain systems of basic axioms, i.e. in systems of logically complex 'sentences'. Others think that formal truth could be defined axiomatically. We should differentiate, here, between

1) a definition of formal satisfaction or truth conditions for a system of sentences resp. of a concrete structure being a possible model for classes of formulas or formal sentences,

2) a proof that such a structure is a model for a class of formulas, i.e. that the formulas are turned into true sentences by an 'interpretation',

3) a proof of formal (deductive) consistency of a system of formal axioms or a proof of the 'classical' existence of models for the formulas, and

4) a mere 'belief' in consistency of such a system or in the classical existence of models in a vague realm of possible models.

If a system of axioms is seen as (a part of) a 'definition' of a model or a class of possible models, we do not 'believe' in the axioms. We might believe 
in its consistency, if we do not have a sufficient proof or argument for it. But neither the axioms of Peano arithmetics, nor of geometry, nor of set theory really can define arithmetics, geometry or set theoretical truths - whatever Hilbert believed neglecting Frege's (correct) criticism. We can (and must) show that the formal axioms of such a theory articulate 'true' statements and 'true' rules with respect to 'elementary' arithmetics, geometry or set theory, where the concept of truth is defined by certain non-axiomatic conditions. I do not have the space here to say more about this rather far-reaching claim. As an example, it suffices to show that it might be a reasonable (or even true) claim.

If we consider the role played by true statements in wider contexts of judgements, practice and actions, we may see that pragmatists rightly claimed that truth, not only mathematical truth, is a formal concept. The same holds for the concept of an object (or 'entity') relative to a category $\boldsymbol{R}$ : It is, as I hope to have shown, at least at first always a totally formal concept.

The result of this insight is, if I am right: When we want to know how we use the forms of speech considered here and the corresponding formal terminological and logical rules, we have to experiences, goals and interests, practical successes and certainties into our picture. Only if our interest remains restricted to an analysis of formal features of meaning, i.e. of formal truth-values and formal truth-conditions of sentences (or statements), as Frege was, we may 'abstract' from possible 'external' roles played by the concept of truth and from how we relate the formal realms of 'internal' objects we talk about to external experience and practice. Any such 'abstraction' refrains from an analysis of concrete and complete meaning.

Wittgenstein and before him William James noticed that a Russellian and Fregean analysis of sense, meaning and truth remains abstract and formalistic in this sense. Indeed, truth conditional semantics as it was developed by Frege, Tarski, Davidson or Montague remains 'internalistic', i.e. it has only a limited interest, just like Frege's differentiation between sense and meaning does not suffice (at all) for a 'whole' analysis of meaning.

But let us come back to our title question: What is a (meaningful) name? The result of our consideration should be this: The see mingly obvious answer: „A (meaningful) name is a sign or symbol that is used to name an object" does not suffice at all. We cannot give a sufficient answer to this question without explaining what it is to be an object that can be named. For this, we want to know what it is to name an object. I take it that it was these questions Frege tried to answer. And he noticed that we cannot answer it without reference to a whole system of sentences. 
Because we can arrive at this more sophisticated analysis just by reading Frege, it seems to be a matter of charity to give him the credit and not to assume that we 'improved' his allegedly erroneous 'Platonism' - even if we might be successfull in removing some more or less important errors and ambiguities of his presentation.

Pirmin Stekeler-Weithofer

Institut für Philosophie

Universität Leipzig

Augustusplatz 9

04109 Leipzig, Germany 